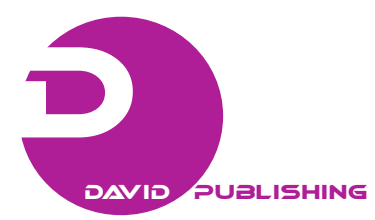

\title{
Diabetes Mellitus: Too Many Controversies Affecting
}

\section{Care}

\author{
Anil Kumar Mandal ${ }^{1}$ and Linda Marie Hiebert ${ }^{2}$
}

1. Department of Medicine, University of Florida, Gainesville 32611, Florida, USA

2. Department of Veterinary Biomedical Sciences, University of Saskatchewan, Saskatoon S7N 5B4, Canada

\begin{abstract}
Diabetes care is in disarray mainly due to a lack of consensus among professionals in the diagnosis and management of diabetes. The most common trend is the use of $\mathrm{HbA}_{1} \mathrm{c}$ (glycosylated hemoglobin) above $6.5 \%$ in making the diagnosis of diabetes. Similarly, the most common prescription written for treatment of diabetes is metformin, $500 \mathrm{mg}$ to $1000 \mathrm{mg}$ twice daily. FBG (Fasting blood glucose) above $126 \mathrm{mg} / \mathrm{dL}$ is a common accompaniment of $\mathrm{HbA}_{1} \mathrm{c}$, but 2hPPG (2-h postprandial glucose) or 4-h oral glucose tolerance tests are not included in the diagnosis of diabetes. In the early stage of diabetes or impaired glucose tolerance associated with obesity, $\mathrm{FBG}$ or $\mathrm{HbA}_{1} \mathrm{c}$ may be useful, but with rising $\mathrm{FBG}$ or $\mathrm{HbA}_{1} \mathrm{c}$ despite dual or triple oral antidiabetic therapy, $2 \mathrm{hPPPG}$ becomes the mainstay to determine the severity of diabetes and the need for initiation of insulin therapy. It is needless to underscore that $2 \mathrm{hPPG}$ relates to outcome measures such as cardiovascular disorders, nephropathy and death. Thus the cornerstone of therapy when $2 \mathrm{hPPG}$ is above $200 \mathrm{mg} / \mathrm{dL}$ ( $\geq 11.1 \mathrm{mmol} / \mathrm{L}$ ) is insulin with a goal to reduce the risk of complications. There is no or only subtle evidence that oral antidiabetic agents will affect $2 \mathrm{hPPG}$ or reduce the risk of complications of diabetes. Therefore, the optimal goal of therapy in diabetes is not limited to merely glycemic control, but the intent of glycemic control which is prevention of diabetes complications.
\end{abstract}

Key words: Diabetes mellitus, $2 \mathrm{~h}$ postprandial glucose, insulin therapy.

\section{Introduction}

Diabetes mellitus or diabetes is the biggest challenge in current medical practice. Challenge means utmost attention is required by the professionals for the most important task in diabetes care, which is prevention of complications of diabetes, namely: blindness, amputation of toes or legs, kidney failure and dialysis, heart attack and sexual dysfunction. Cure of diabetes is far-fetched, but prevention of the complications is attainable. Prevention of the complications is also essential because they are disabling and the cost involved to treat the complications is enormous. Importantly, this enormous cost of treating diabetes complications is imposing an excessive burden economically on the society worldwide.

Corresponding author: Anil Kumar Mandal, M.B., B.S., professor, research fields: diabetes and hypertension. E-mail: amandal@med-spec.com.
Diabetes, like high blood pressure, is a silent disease - in particular, in adults - and the damage caused to the organs by high glucose levels is an insidious process. Thus the damage remains undetected for a long time and manifests severely in late stage. The damage caused by high glucose to the organs is fairly uniform, both in children and young adults, and in older adults and the elderly. However, symptoms of diabetes can be explosive in children and young adults due to polyuria (visiting bathroom every few minutes), polydipsia (excessive thirst), and rapid loss of weight. On the other hand, manifestations can be very subtle in older adults. Adults often do not know they have diabetes until symptoms manifest due to the damage to an organ. Some of the symptoms, not in any order, are urinary retention, inability to obtain penile erection, decreased vision, chest pain, protein in urine, or decreased kidney function found on routine physical examination, recurrent urinary tract infection, non-healing foot ulcer, or discoloration of a foot or 
toes. In fact, diabetes is often detected for the first time in patients brought into the emergency room of a hospital for chest pain.

\section{Controversies Affecting Care}

The controversies concern diabetic nephropathy versus other complications of diabetes. Numerous studies in the past have confirmed that tight glycemic control with multiple insulin injections prevent microvascular and macrovascular complications of diabetes [1-5]. From these studies, it is evident that glycemia above the normal range of glucose (70-99 $\mathrm{mg} / \mathrm{dL} \quad(3.8-5.5 \mathrm{mmol} / \mathrm{L})$ can be injurious to microvascular system. Since high glucose bathes the entire vascular system involving all organs, the injury is likely to be uniform in all organs. Our cell culture studies using normal glucose of $90 \mathrm{mg} / \mathrm{dL}$ ( $5 \mathrm{mmol} / \mathrm{L}$ ) and high glucose of $540 \mathrm{mg} / \mathrm{dL}(30 \mathrm{mmol} / \mathrm{L})$ by treating vascular endothelial cells show no damage or severe damage, respectively [6]. Our cell culture studies also revealed that addition of insulin along with high concentration of glucose abrogated the damage. Thus we developed a hypothesis from our cell culture studies which is as follows:

Damaged endothelial cells shed off into the $\downarrow$

Capillary lumen

$\downarrow$

Calcification of necrotic tissue with occlusion of capillary lumina

$\downarrow$

Ischemia of the organ.

This is a slow ischemia giving rise to fibrosis. In the kidney, glomerular sclerosis and interstitial fibrosis give rise to chronic renal failure and end stage renal disease. In the heart, coronary artery occlusion gives rise to angina. In the extremities, foot ulcer and gangrene supervene. In the retina, microaneurism is formed. Therefore our cell culture studies have permitted us to develop a unified theory which is similar to other authors [7] to explain all diabetic complications. Therefore, if we can agree with unified theory, our focus will be directed to control hyperglycemia to mitigate the complications.

It is intriguing to think why glucose at a high level-for instance, $\geq 200 \mathrm{mg} / \mathrm{dL} \quad(\geq 11.1$ $\mathrm{mmol} / \mathrm{L}$ ) — causes damage to the organs, whereas the similar glucose at a normal level does not. Therefore, an important question can be asked: If glucose, in and of itself, has any direct effect in causing the tissue damage or whether a high level of glucose does so by altering cellular functions. To answer that question, many theories were proposed and tested but not applied. However, a plausible mechanism of cellular injury is inhibition of glutathione pathway by high glucose environment. Excessive number of oxygen-free radicals produced by reduced glutathione could play an important role in widespread organ damage in diabetes [8]. In our laboratory, cultured endothelial cells were treated with an inhibitor of glutathione, buthionine sulfoxamine, which showed endothelial cell damage similar to that induced by high glucose [9], thereby supporting glutathione inhibition theory in the pathogenesis of glucose-induced endothelial cell damage.

Although the exact mechanism with regard to organ damage caused by high glucose levels is yet to be unraveled; as of now, we know for sure that sustained elevation of blood glucose level $\geq 200 \mathrm{mg} / \mathrm{dL}$ ( $\geq 11.1$ $\mathrm{mmol} / \mathrm{L}$ ) unequivocally causes organ damage producing a gamut of complications. These complications, which were stated earlier, are seen every day in doctors' offices and hospitals; and they are increasing in numbers. Why are these complications seen in increasing numbers? Because too many controversies surround the treatment of the fundamental pathophysiology of diabetes, which is the uncontrolled hyperglycemia.

\section{Distortion in the Paradigm of Diabetes Therapy}

Diabetes is defined by elevation of blood glucose higher than $200 \mathrm{mg} / \mathrm{dL}(\geq 11.1 \mathrm{mmol} / \mathrm{L})$ in a post-meal glucose challenge or oral glucose tolerance 
test [10], and is associated with deficient insulin production or deficient insulin response to glucose challenge. Thus the logical treatment of diabetes is insulin rejuvenation. But that is not happening because of the distortion in the method of treatment of diabetes. There are currently two forms of this distortion in diabetes management, resulting in increased morbidity and mortality in diabetes.

Distortion \#1. Prevalence of oral antidiabetic agents. As soon as diagnosis of diabetes is made in the office or clinic, the typical sequence of events is a prescription of metformin $500 \mathrm{mg}$ PO twice daily increasing to $1,000 \mathrm{mg}$ twice daily if the FBG (fasting blood glucose) is not brought under control or more commonly, if HbA1c (glycosylated hemoglobin) is not less than $7 \%$. Thereafter, glyburide, $10 \mathrm{mg}$ PO twice daily or pioglitazone $15-30 \mathrm{mg}$ twice daily is added to promote glycemic control. Now the incretin is on the horizon. A common prescription is a DPP-4 inhibitor. When the glucose levels are still elevated despite dual or triple therapy and/or the patient develops a complication such as foot ulcer, chest pain suggestive of coronary artery disease, or decreased kidney function, they are advised to start an insulin therapy, most commonly a nightly dose of Glargine insulin.

An important question is why is the insulin initiated so late in the treatment? This was the topic of a symposium in the 4th World Congress of Controversies in CODHy (Consensus in Diabetes, Obesity and Hypertension) held in Barcelona, Spain during November of 2012.

Distortion \#2. There are several factors in this distortion which impair glucose control and increase the risk of developing complications. These factors are:

(1) Gaps in knowledge of which glycemic parameters is important in achieving glycemic control with a goal to prevent diabetic complications. There is no consensus in the validity of FBG, 2hPPG (2-h postprandial blood glucose), and $\mathrm{HbA}_{1} \mathrm{c}$ in glycemic control [11];

(2) Most studies have used $\mathrm{HbA}_{1} \mathrm{c}$ to reveal glycemic control but did not focus on outcome measures;

(3) Outcome measures have focused on cardiovascular disorders consisting of myocardial infarction or death associated with $2 \mathrm{hPPG} \geq 200$ $\mathrm{mg} / \mathrm{dL}$ [12-13]. Even DECODE study has shown that risk of cardiovascular death increases threefold as 2-h post challenge glucose levels increase from $54 \mathrm{mg} / \mathrm{dL}$ to $199 \mathrm{mg} / \mathrm{dL}$, although these readings are in the non-diabetic range (Fig. 1);

(4) There is a paucity of data on the relationship between $2 \mathrm{hPPG}$ and renal function changes. However, a single study from Italy confirmed that $2 \mathrm{hPPG}$ greater than $200 \mathrm{mg} / \mathrm{dL}$ and $\mathrm{HbA}_{1} \mathrm{c}$ above $8 \%$ (established diabetes) is closely linked to rapid decrease in GFR, whereas a 2hPPG of less than 200 $\mathrm{mg} / \mathrm{dL}$ and $\mathrm{HbA}_{1} \mathrm{c}$ below $8 \%$ is associated with trivial or no change in GFR [14]. Our data are consistent with the report from Italy, and in addition our data show that there is a poor correlation between $\mathrm{HbA}_{1} \mathrm{c}$ and renal function parameters [15]. Other studies have shown that reduced $2 \mathrm{hPPG}$ is strongly associated with reduction in retinopathy and nephropathy [16].

Despite the great importance of $2 \mathrm{hPPG}$ asserted in the outcome measures of cardiovascular disorders, and albeit some importance in renal failure, $\mathrm{HbA}_{1} \mathrm{c}$ continues as the standard test for evaluation of diabetes control in most studies whether in clinics or offices.

An apparent reason for this solidarity is a commercial bias because most oral antidiabetic agents reduce $\mathrm{HbA}_{1} \mathrm{c}$ [17]. There is slight, or no evidence that oral antidiabetic agents affect $2 \mathrm{hPPG}$;

(5) Occasional studies showed that use of metformin alone or metformin in combination with insulin in Type 2 diabetes reduced the risk of myocardial infarction [18]. However, this finding was refuted by another study wherein 390 patients treated with insulin were assigned to randomly receive 


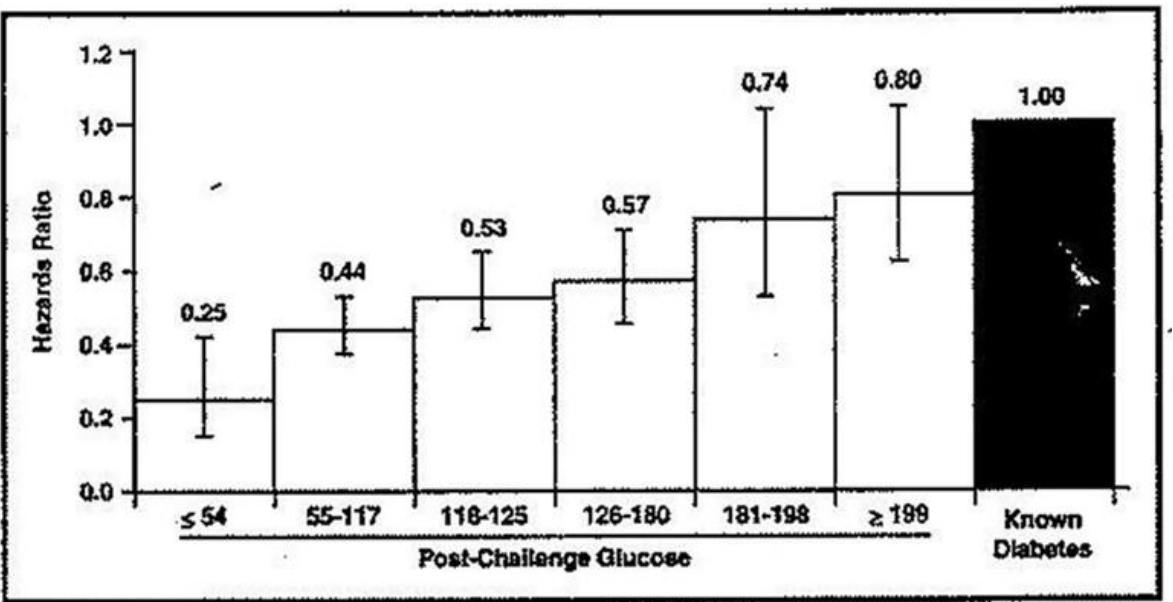

Fig. 1 DECODE Study Group. Is the current definition of diabetes relevant to mortality risk from all causes and cardiovascular and non-cardiovascular disease?

The risk for cardiovascular death increases threefold as $2 \mathrm{~h}$ post-challenge glucose levels increase from $54 \mathrm{mg} / \mathrm{dL}$ to 199 $\mathrm{mg} / \mathrm{dL}$, although these readings are all in the non-diabetic range. Data were adjusted for age, gender, weight, systolic blood pressure, cholesterol, and smoking during the 11 years of follow-up for 29,712 patients in the DECODE (Diabetes Epidemiology: Collaborative Analysis of Diagnostic Criteria in Europe) study.

metformin or placebo. The primary endpoints were aggregate of microvascular and macrovascular morbidity and mortality. Metformin improved glycemic control and reduced insulin requirements but did not reduce primary endpoints [19].

\section{Major Distortion and Worst Controversy in Therapy of Diabetes}

\subsection{Treatment of Proteinuria}

A major distortion and the worst controversy lie in the management of diabetic nephropathy and renal failure. Diabetic nephropathy is manifested by no to heavy proteinuria depending on the duration of the history of diabetes, adequacy of glycemic controls, and therapy used to achieve glycemic controls. In general, the longer the duration of uncontrolled hyperglycemia, in particular glycemic level consistently above $200 \mathrm{mg} / \mathrm{dL}$ ( $\geq 11.1 \mathrm{mmol} / \mathrm{L}$ ), and no insulin rejuvenation, the more likely heavy proteinuria will be found. Most commonly diabetes patients reveal $1+$ to $2+$ dipstick proteinuria or quantitatively less than $500 \mathrm{mg} 24 \mathrm{~h}$ proteinuria. This mild to moderate level of proteinuria has slight or no adverse effect on renal function [20].
However, a previous study swung the pendulum from glycemic control to minimal proteinuria (microalbuminuria) control in diabetes. This rat study showed that hyperglycemia causes hyperfiltration, and hyperfiltration is associated with microalbuminuria and glomerulosclerosis. They hypothesized that by reducing microalbuminuria with ACEI (angiotensin converting enzyme inhibitors), glomerulosclerosis or diabetic nephropathy will be mitigated [21]. Thereafter a clinical study was done in which diabetes patients were treated with the ACEI captopril and found doubling of serum creatinine in a fewer captopril treated versus the control group. The $\mathrm{HbA}_{1} \mathrm{c}$ levels were above $11 \%$ in both groups. $2 \mathrm{hPPG}$ levels were not provided and whether these patients were treated with insulin was not stated. Thus this study showed fewer captopril treated patients developing ESRD (End Stage Renal Disease) revolutionized microalbuminuria as a standard test for diabetes and therapy with ACEI drugs is a standard of care in diabetes [22].

In the current day practice, glucose control, which is fundamental to prevention strategies, has taken the back seat; whereas, ACEI and ARB (angiotensin 
receptor blocker) drugs, have taken the forefront in the marketplace. Given the widespread consensus of ACEI/ARB as the renoprotective drugs, insulin rejuvenation is considered an old fashioned therapy. This trapped position of glucose control-combined with the deceptive consensus of ACEI/ARB as a frontier therapy of diabetes - has resulted in increased incidence of amputation, ESRD and opening of growing numbers of private dialysis clinics to treat the ESRD patients. All these are lucrative for the professionals and the industry, but tragic for the patients and their families.

Most patients who develop ESRD are automatically started on dialysis therapy, even though they are asymptomatic and exhibit no fluid, electrolytes or acid-base disorders. Maintenance dialysis therapy prolongs survival, but this survival is less than optimal associated with more time in hospitals than at home [23], and the expenditures from taxpayer financed programs and personal insurance are far out of proportion to the quality of survival. For instance, dialysis therapy in the USA is exhausting Medicare and Medicaid budgets, leaving fewer dollars for the prevention strategies. Further, progressive physical burden and mental anguish imposed on the loved ones and the demands of in-home care associated with pre- and post-dialysis treatments are detrimental to the family. Combining all of these adverse outcomes, a serious question can be raised about the overall benefit of chronic dialysis therapy in diabetes [23].

On the other hand, ESRD is largely preventable by adequate control of hyperglycemia (glucose level of < $200 \mathrm{mg} / \mathrm{dL}$ or $<11.1 \mathrm{mmol} / \mathrm{L}$ ) by intensive insulin therapy implemented early in the course of diabetes and control of hypertension by antihypertensive therapy without the use of ACEI/ARB drugs. It is needless to underscore that ACEI/ARB drugs are not renoprotective [24].

\subsection{Therapy by Oral Antidiabetic Agents}

Oral antidiabetic agents are good in lowering fasting glucose but practically ineffective in lowering postprandial glucose surge. At least, no evidence exists to that effect. Postprandial glucose surge does not necessarily cause any unusual symptoms immediately, thus not alerting the patients or the professionals to take any action. However, patients who home monitor the glucose levels regularly find out that their glucose is not under control with oral antidiabetic drugs which leads to emergency rooms visits, or consultations with another doctor to get advice for a different, albeit better treatment, for good glucose control.

Since postprandial glycemic surge does not produce symptoms and the fact that patients refuse to take insulin because of fear of injections, professionals and pharmaceutical companies are at an advantageous position to exploit the ignorance of the patients with regard to the seriousness of uncontrolled hyperglycemia. Hence, it has become a standard practice to prescribe multiple oral antidiabetic agents to keep HbA1c at $6.5 \%$ or less [25] thereby making the patients happy with a false sense of security, and return again and again to their doctors' offices. Preaching treatment with insulin again and again carries a distinct risk for the doctors of losing the patients, and hence the income.

What can we do to overturn the current practice of diabetes care and treat patients appropriately to ensure a successful journey to control of diabetes and not a crash, somewhere in the middle of the trip, as is happening now with amputation, ESRD or heart attack? Here is one idea to that effect: Patients who have understood that good glucose control with insulin is permissive of a healthy life can be considered as a significant part of prevention strategies. Therefore, prevention strategies comprise:

(1) Group therapy through volunteerism of insulin-treated patients who are doing well for other patients who have refused home monitoring of glucose and to take insulin injections. It will be an innovative step in order to increase the number of patients to take insulin and keep glucose under control. 
It is like a clinical trial except diabetic patients who are taking insulin will act as counselors to the patients who have refused to take insulin to make them understand how uncontrolled diabetes can jeopardize their life and how well-controlled diabetes with insulin therapy can permit them to live a healthy life. The volunteerism is attainable and will be the least expensive, albeit the most profitable, part of diabetes care. This approach is also likely to reduce the rate of intentional insulin omission [26];

(2) Adequate glucose control by prescribed insulin therapy consisting of insulin Glargine or detemir after breakfast and dinner and regular insulin before each meal to reduce post-meal excursions [27];

(3) Adequate control of hypertension by antihypertensive drug therapy singly or in combination, for example, beta blocker, calcium channel blocker and vasodilators. They are safe and effective;

(4) Avoidance of ACEI/ARB drugs. It is fundamentally wrong to prescribe these drugs as renoprotective drugs and ignore glucose control, which is critical in prevention strategy;

(5) Shift focus away from microalbuminuria control and give full attention to a total care of the diabetic patient with details to bladder function, foot care, sexual function, cardiovascular health, cognitive function and finally overall quality of life;

(6) Frequent office visits are an integral part of total diabetes care, as stated above. Diabetes patients often have the illusion of going into dialysis. Professionals must counsel the patients to invalidate such illusion. Dialysis should be considered as an accident and not a part of routine diabetes therapy.

\section{Conclusions}

It is evident from the preponderance of literature that more attention is paid to $\mathrm{HbA}_{1} \mathrm{c}$ levels than to any other parameter related to diabetes control. This is regrettable as our own studies and many others have shown that when 2-hour postprandial glucose is used as the leading measurement for glycemic control in conjunction with insulin injection treatment over oral antidiabetic agents, the outcome measurements are greatly improved. Renal function in particular remains stable and in many cases, is improved [27]. Cardiovascular disorders may also be forestalled with use of insulin therapy over oral antidiabetic drugs [18].

Perhaps the most important point to make in this commentary is that the physicians and the patients must work hand in hand. It is the physician's responsibility to encourage insulin therapy either when diabetes is first diagnosed or promptly when oral therapy fails, thereby avoiding beta cell exhaustion and death [28]. This will ensure stability of metabolic control and reduce complications related to metabolic abnormalities, which will translate into improved overall health, improved quality of life with decreased comorbidities, and a longer life expectancy [28].

\section{References}

[1] P. Reichard, B. Berglund, A. Britz, I. Cars, B.Y. Nilsson, U. Rosenqvist, Intensified conventional insulin treatment retards the microvascular complications of IDDIABETES (insulin-dependent diabetes mellitus): The SDIS (Stockholm Diabetes Intervention Study) after 5 years, J. Intern. Med. 230 (2) (1991) 101-108.

[2] C.P. Soper, J.L. Barron, S.L. Hyer, Long-term glycemic control directly correlates with glomerular filtration rate in early type 1 diabetes mellitus before the onset of microalbuminuria, Diabet. Med. 15 (12) (1998) 1010-1014.

[3] J. Fabre, L.P. Balant, P.G. Dayer, H.M. Fox, A.T. Vernet, The kidney in maturity onset diabetes mellitus: A clinical study of 510 patients, Kidney Int. 21 (5) (1982) 730-738.

[4] The Diabetes Control and Complications Trial Research Group, The effect of intensive treatment of diabetes on the development and progression of long-term complications in insulin-dependent diabetes mellitus, $\mathrm{N}$. Engl. J. Med. 329 (1993) 977-986.

[5] UK Prospective Diabetes Study Group 33, Intensive blood-glucose control with sulphonylureas or insulin compared with conventional treatment and risk of complications in patients with type 2 diabetes, Lancet 352 (1998) 837-853.

[6] A.K. Mandal, J.T. Puchalski, S. Lemley-Gillespie, C.A. 
Taylor, M. Kohna, Effect of insulin and heparin on glucose-induced vascular damage in cell culture, Kidney Int. 57 (2000) 2492-2501.

[7] M. Brownlee, Pathobiology of diabetic complications: A unifying mechanism, Diabetes 54 (2005) 1615-1625.

[8] L.M. Hiebert, J. Liu, Heparin protects cultured arterial endothelial cells from damage by toxic oxygen metabolites, Atherosclerosis 83 (1990) 47-51.

[9] A.K. Mandal, T. Ping, S.J. Caldwell, R. Bagnell, L.M. Hiebert, Electron microscopic analysis of glucose-induced endothelial damage in primary culture: Possible mechanism and prevention, Histol. Histopathol. 21 (2006) 941-950.

[10] M.I. Harris, Undiagnosed NIDDM clinical and public health issues, Diabetes Care 16 (1993) 642-652.

[11] A.K. Mandal, L.M. Hiebert, Dilemma in diabetes care relates to its complications: Strategies for prevention, J. Diab. Res. Clin. Met. 11 (2012), available online at: http://www.hoajonline.com/jdrcm/2050-0866/1/15 (accessed Oct. 15, 2005)

[12] J.E. Gerich, Postprandial HYPErglycemia and cardiovascular disease, Endo. Pract. 12 (Suppl) (2006) 47-51.

[13] A.V. Aryangat, J.E. Gerich, Type 2 diabetes: Postprandial hyperglycemia and increased cardiovascular risk, Vascular Health and Risk Management 6 (2010) 145-155.

[14] R. Nosadini, G. Tonolo, Relationship between blood glucose control, pathogenesis and progression of diabetic nephropathy, J. Am. Soc. Nephrol. 15 (Suppl) (2004) S1-S5

[15] A.K. Mandal, L.M. Hiebert, H. Khamis, Dglucose is linked to renal function changes in diabetes, Diab Res ClinPract 91 (2011) 191-194.

[16] D.R. McCance, R.L. Hanson, M.A. Charles, L.T. Jacobsson, D.J. Pettitt, P.H. Bennett, et al., Comparison of tests for glycated hemoglobin and fasting and two hour plasma glucose concentrations as diagnostic methods for diabetes, BMJ 308 (1994) 1323-1328.

[17] S.C. Liu, Y.K. Tu, M.N. Chien, K.L. Chien, Effect of antidiabetic agents added to metformin on glycemic control, hypoglycemia and weight change in patients with Type 2 diabetes: A network meta-analysis, Diab. Obes. Metab. 14 (2012) 810-820.
[18] R.R. Holman, S.K. Pal, M.A. Bethel, D.R. Mathews, H. A. Neil, 10-year follow up of intensive glucose control in Type 2 diabetes, N. Engl. J. Med. 359 (2008) 1577-1589.

[19] A. Kooy, J. de Jager, P. Lehert, D. Bets, M. G. Wulffele, A.J.M. Donker, et al., Long-term effects of metformin on metabolism and microvascular and macrovascular disease in patients with Type 2 diabetes mellitus, Arch. Intern. Med. 169 (2009) 616-625.

[20] P.D. Springberg, LE.Jr. Carrett, AL.Jr. Thompson, N.F. Collins, R.E. Lordon, R.R. Robinson, Fixed and reproducible orthostatic proteinuria: Results of a 20-year follow up study, Ann. Intern. Med. 97 (1982) 516-519

[21] R. Zatz, B.R. Dunn, T.W. Meyer, S. Anderson, H.G. Rennke, B.M. Brenner, Prevention of diabetic glomerulopathy by pharmacologic amelioration of glomerular capillary hypertension, J. Clin. Invest. 77 (1986) 1925-1930.

[22] E.J. Lewis, L.G. Hunsicker, R.P. Bain, R.D. Rohde, The effect of angiotensin-converting enzyme inhibition on diabetic nephropathy, N. Engl. J. Med. 329 (1993) 1456-1462.

[23] J.R. Berger, S. Hedayati, Renal replacement therapy in the elderly population, Clin. J. Am. Soc. Nephrol. 7 (2012) 1039-1046.

[24] A.K. Mandal, L.M. Hiebert, Renal protection in diabetes: Is it affected by glucose control or inhibition of the renin-angiotensin pathway, Clin. Nephrol. 69 (2008) 169-178.

[25] H.W. Rodbard, P.S. Jellinger, J.A. Davidson, D. Einhorn, A.J. Garber, G. Grunberger, et al., Statement by and american association of clinical endocrinologists american college of endocrinology consensus panel on type 1 and type 2 diabetes mellitus: An algorithm for glycemic control, Endocr. Pract. 15 (2009) 540-559.

[26] K. Weinger, E.A. Beverly, Barrier to achieving glycemic targets: Who omits insulin and why, Diabetes Care 33 (2010) 450-452.

[27] A.K. Mandal, N.S. Neki, Diabetes: Pragmatic therapy with a goal to prevent end stage kidney disease and dialysis, Open Jour. Int. Med. 1 (2011) 80-92.

[28] P.J. Palumbo, The case for insulin treatment early in type 2 diabetes, Clev. Clin. J. Med. 71 (2004) 385-405. 